\title{
An NMR Spin-Echo Study of Diffusion in a Room-Temperature Molten Salt
}

\author{
Günter Palmer, Joachim Richter, and Manfred D. Zeidler \\ Institut für Physikalische Chemie, Rheinisch-Westfälische Technische Hochschule Aachen, \\ Templergraben 59, 52056 Aachen, Germany \\ Reprint requests to Prof. J. R.; Fax: 0049(0)2418092235; e-mail: richter@rwth-aachen.de
}

Z. Naturforsch. 59a, 59-63 (2004); received November 24, 2003

Pressure-dependent measurements of self-diffusion in the room-temperature molten salt (RTMS) N,N-butylmethylimidazoliumhexafluorophosphate were carried out at 298 and $308 \mathrm{~K}$. The pressure range lied between ambient pressure and $300 \mathrm{MPa}$. In addition, methanolic solutions of this RTMS were investigated at ambient pressure. The self-diffusion coefficients of both components are reported as functions of concentration and temperature in the range 293-313 K. A modified version of a recently published high-pressure probe is described.

Key words: Room-temperature Molten Salts; Self-diffusion; NMR Spin Echo.

\section{Introduction}

Recently room-temperature molten salts (RTMS's) became important, for example as substitutes for organic solvents in industrial processes [1-5]. The characterization of RTMS's is still in its initial stage, and a relation between their structure and properties is far from being known. Self-diffusion coefficients are scarcely available for RTMS's [6], and thus we undertook a study of the self-diffusion of N,N-butylmethylimidazolium hexafluoro-phosphate $(\mathrm{BMIM}) \mathrm{PF}_{6}$ at different conditions.

We modified a recently constructed high-pressure probe head [7] which can be used at pressures up to $200 \mathrm{MPa}$ at $613 \mathrm{~K}$, to operate in the temperature range 293 to $323 \mathrm{~K}$. Due to the low temperature needed for the RTMS's, the titanium alloy IMI 834, from which the high-pressure cell was constructed, allowed pressures up to $300 \mathrm{MPa}$.

\section{Experimental}

The new probe head had to fit the available Bruker CXP $300 \mathrm{nmr}$ spectrometer with a magnet having an inner bore of $72 \mathrm{~mm}$ within the shim coils at a field of $7.02 \mathrm{~T}$.

Figure 1 shows the probe head. From the right we see the high-pressure cylinder made of the titanium al- loy IMI 834, as already described in [7], followed by an aluminum tube with grooves and a cover of polyoxymethylene. The $141 \mathrm{~mm}$ long and $3 \mathrm{~mm}$ thick aluminum tube has an inner diameter of $60 \mathrm{~mm}$ and thus fits tightly the high-pressure cylinder. The grooves are $6 \mathrm{~mm}$ wide, $1.5 \mathrm{~mm}$ deep and have an angle of inclination of $3.47^{\circ}$. Air of the desired sample temperature is pushed through the grooves from below. This setup fits into the magnet bore including the shim coils, and no further cooling jacket is required since the shim coils can withstand temperatures of $323 \mathrm{~K}$. All other parts of the high-pressure cell are the same as before.

The high-frequency coil and the circuit had to be adjusted to $300 \mathrm{MHz}$ for measurement of the proton NMR frequency in the BMIM cation. At such a high frequency stray inductivities and capacities are much more disturbing and must be excluded by short leads. The compact circuit is shown in Figure 2.

As pressurizing fluid we used hexadecafluoroheptane (3M Company, Neuss, Germany, type FC 84). Pressure is applied through a screw press and leads to the probe head by a capillary.

The gradient coil, together with the current unit and readout unit, were taken from the previous publications $[7,8]$. The gradient was $0.044 \mathrm{Tm}^{-1} \mathrm{~A}^{-1}$. The sample temperature, pressure and gradient current are recorded and fed through an $\mathrm{AD}$ converter [7] into a computer, so that any failure can be traced. 


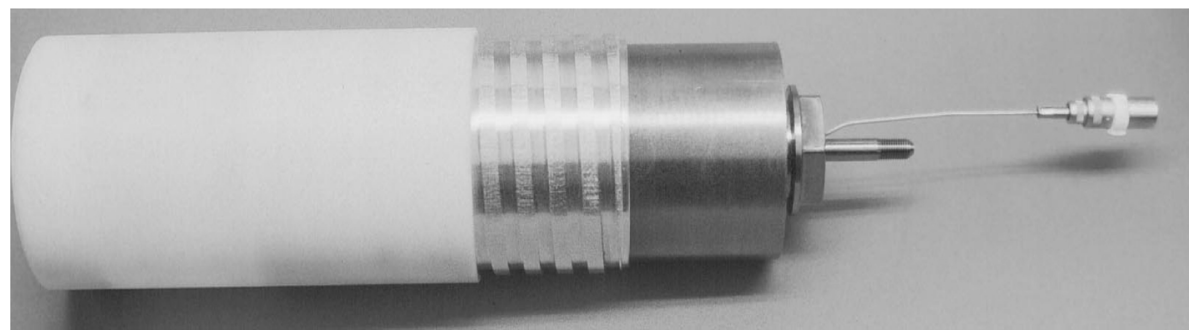

Fig. 1. Probe head.

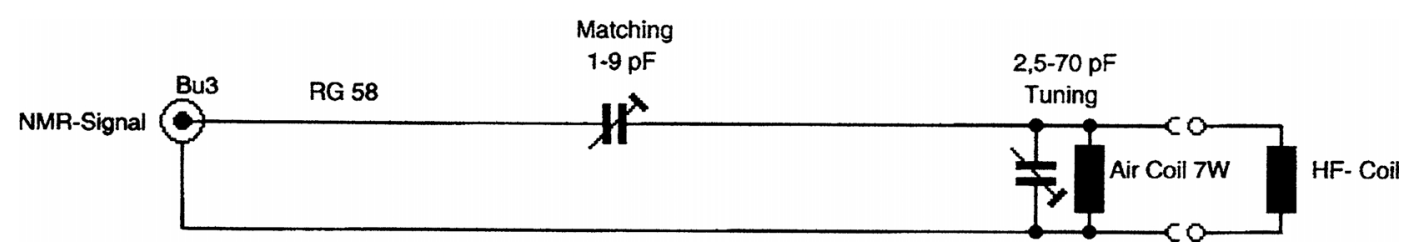

Fig. 2. Resonance circuit.

Table 1. Fit parameters to the concentration dependence of the self-diffusion coefficient of the BMIM cation (B) and of methanol (M) in methanolic solution at different temperatures.

\begin{tabular}{llccccc}
\hline$T / \mathrm{K}$ & & 293 & 298 & 303 & 308 & 313 \\
\hline$D_{0} /\left(10^{-9} \mathrm{~m}^{2} \mathrm{~s}^{-1}\right)$ & B & 0 & 0 & 0 & 0 & 0 \\
& M & 0.118 & 0.134 & 0.131 & 0.159 & 0.173 \\
$a /\left(10^{-9} \mathrm{~m}^{2} \mathrm{~s}^{-1}\right)$ & B & 2.824 & 2.881 & 2.895 & 2.901 & 2.819 \\
& M & 2.125 & 2.287 & 2.521 & 2.666 & 2.781 \\
$\mathrm{~b}$ & B & 8.657 & 8.253 & 8.052 & 7.834 & 7.352 \\
& M & 4.104 & 4.025 & 4.056 & 3.979 & 3.861 \\
\hline
\end{tabular}

The self-diffusion coefficient $D$ is determined by the relation

$$
E=\exp \left(-\gamma^{2} g^{2} D \delta^{2}\left[\tau-\frac{\delta}{3}\right]\right)
$$

where $E$ is the echo amplitude, $\tau$ the time in the sequence $90^{\circ}$ pulse- $\tau-180^{\circ}$ pulse- $\tau$-echo, $g$ the gradient strength, and $\delta$ the length of the gradient pulse. The determination of $D$ is done by using varying $\delta$ values, while $g$ is kept constant.

\section{Results and Discussion}

\subsection{Pure (BMIM)PF 6}

Figure 3 shows the self-diffusion coefficients of the BMIM cation between ambient pressure and $300 \mathrm{MPa}$ at 298 and $308 \mathrm{~K}$. The pressure dependence is exponential, and obviously the temperature dependence is dominating. The errors for the diffusion coefficient, the pressure, and the temperature are $3 \%, \pm 0.8 \mathrm{MPa}$, and $\pm 0.2 \mathrm{~K}$, respectively. The activation volumes are $19.1 \mathrm{~cm}^{3} \mathrm{~mol}^{-1}$ at $298 \mathrm{~K}$ and $14.8 \mathrm{~cm}^{3} \mathrm{~mol}^{-1}$ at $308 \mathrm{~K}$.

\subsection{Binary System (BMIM)PF 6 Methanol}

Figures $4 \mathrm{a}$ to $4 \mathrm{e}$ show the self-diffusion coefficients of the BMIM cation and of methanol at different mol fractions and temperatures. The data could be fitted to exponential behavior of the form

$$
D=D_{0}+a \cdot \exp (b x),
$$

the parameters of which being collected in Table 1 . Whereas the diffusion coefficient of methanol decreases by a factor of 10 from the pure liquid to the dilute solution, the corresponding factor is 100 for the BMIM cation. The mutual diffusion coefficient shows a similar behavior [9]. With increasing temperature the two components differ even more.

In spite of the small temperature range we determined the activation energies. They are shown in Figure 5 (p. 62). Maxima appear at the mol fractions 0.7 for methanol and 0.8 for BMIM. For the electrically charged BMIM the activation energy is markedly larger than for the neutral methanol over a large concentration range. However it is still unknown, how much the salt is dissociated in methanol.

\section{Acknowledgement}

Financial support from Fonds der Chemischen Industrie is gratefully acknowledged. We thank Dr. P. Wasserscheid, Institute of Technical Chemistry of the RWTH Aachen, for the preparation of the ionic liquid. 
G. Palmer et al. · An NMR Spin-Echo Study of Diffusion in a Room-Remperature Molten Salt
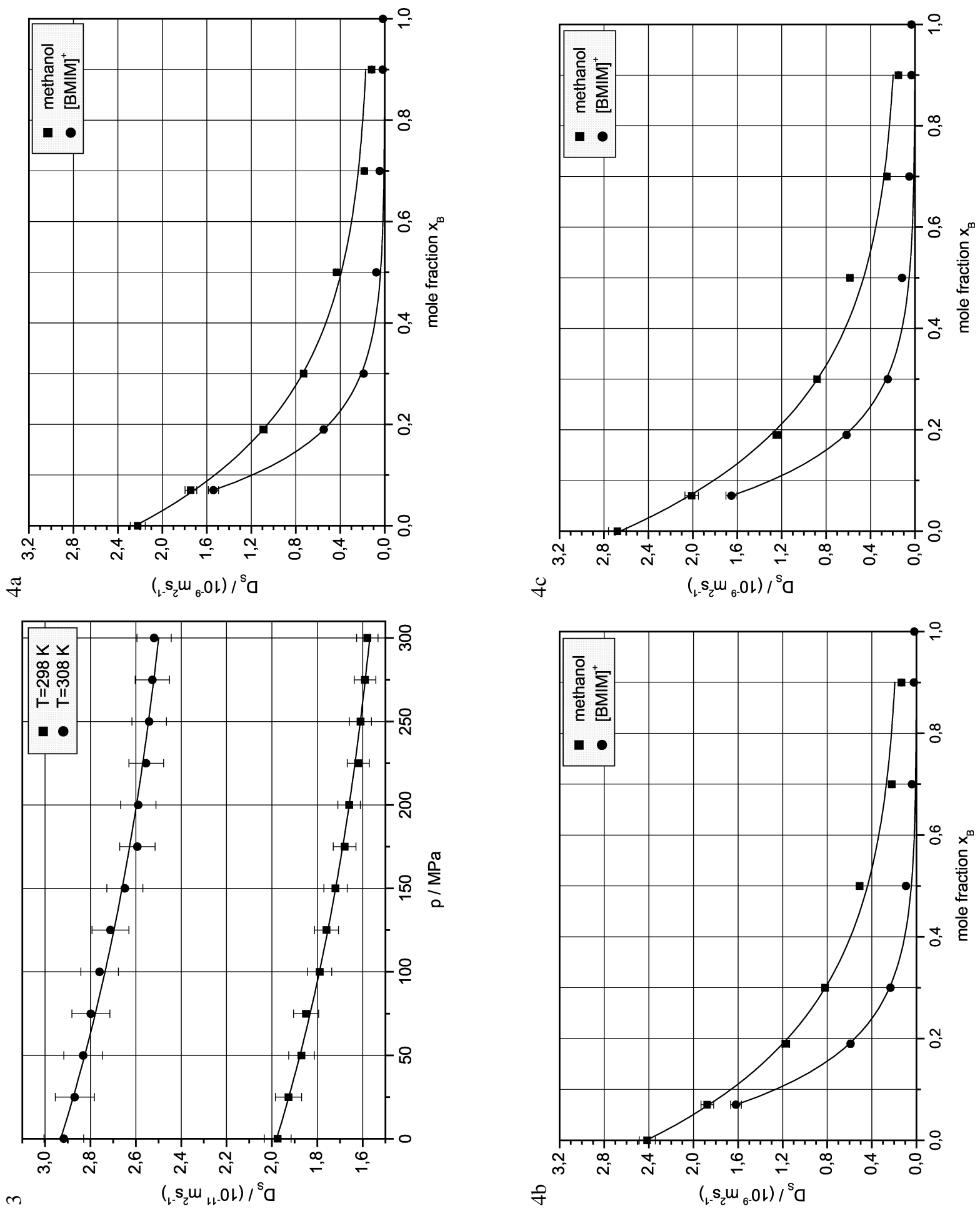

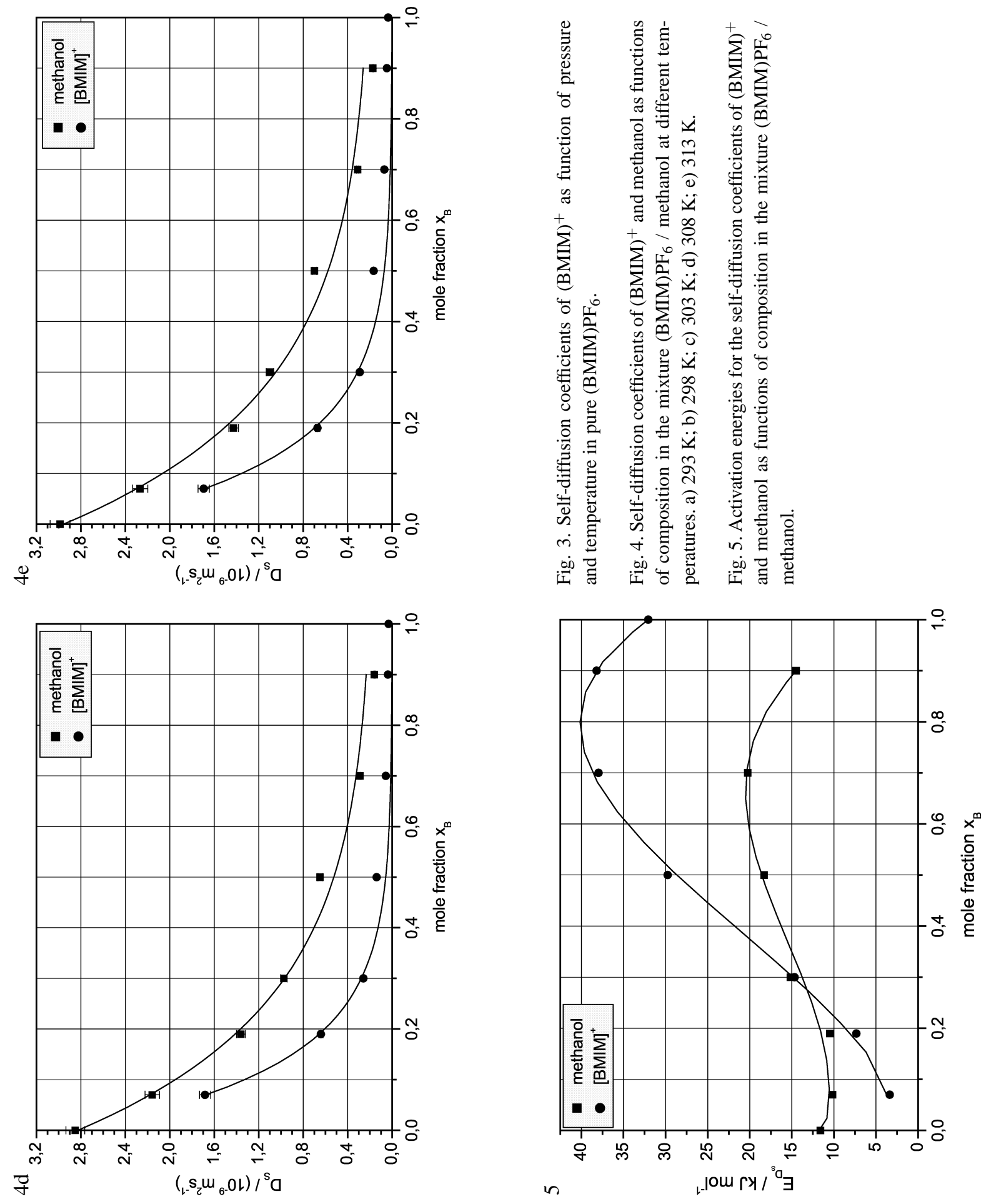
[1] K. R. Seddon, M. J. Earle, and P. B. McCormac, J. Chem. Soc. Chem. Commun. 1998, 2245.

[2] K. R. Seddon and M. J. Earle, Pure Appl. Chem. 72, 1391 (2000).

[3] S. Dai, Y. H. Yu, and C.E. Barnes, J. Chem. Soc. Dalton Trans. 1999, 1201.

[4] J. Xiao, W. Chen, L. Xu, and C. Chatterton, J. Chem. Soc. Chem. Commun. 1999, 1247.

[5] P. Wasserscheid and W. Keim, Angew. Chem. Int. Ed. 39, 3772 (2000).
[6] A. Noda, K. Hayamizu, and M. Watanabe, J. Phys. Chem. B 105, 4603 (2001).

[7] G. Palmer, J. Richter, and M. D. Zeidler, Z. Naturforsch. 59a, 51 (2004).

[8] A. C. Jacob and M.D. Zeidler, Phys. Chem. Chem. Phys. 5, 538 (2003).

[9] A. Leuchter, Dissertation RWTH Aachen, 2002. 